\title{
ENFOQUE Y AVANCES CONCEPTUALES DE LA NUEVA NORMA ESPAÑOLA DE CONSTRUCCIÓN SISMORRESISTENTE NCSE-94
}

\author{
(BASIC PRINCIPLES AND CONCEPTUAL ADVANCES OF THE NEW SPANISH SEISMIC CODE \\ NCSE-94)
}

Rafael Blázquez Martínez, Dr. Ingeniero de Caminos, Canales y Puertos

Dpto. de Ingeniería y Morfología del Terreno(U.P.M.), Presidente de la Asociación Española de Ingeniería Sísmica

ESPAÑA

409-12

\section{RESUMEN}

La plena entrada en vigor, el pasado mes de febrero, de la nueva Norma de Construcción Sismorresistente, tras veinte años de aplicación de la normativa anterior, supone modificaciones conceptuales importantes en todas las fases del diseño antisísmico de edificios, tanto por lo que se refiere al input sísmico como al propio cálculo estructural.

Este cambio de enfoque afecta no sólo a la etapa de proyecto, sino a las de construcción y explotación de la obra y suscita una serie de cuestiones técnicas de gran interés.

En el presente trabajo se analizan estas cuestiones, revisando en profundidad las principales diferencias entre ambas normas, antigua y nueva, así como el grado de convergencia de esta última con el Eurocódigo 8, futura Norma Europea en este campo.

\section{SUMMARY}

The actual substitution of the old Spanish Seismic Code, valid since 1974, by a new regulation means important technical changes which affect every stage of the aseismic design of buildings, all the way from load assessment to structural calculation.

This new approach implies also interesting conceptual changes, not only from the design point of view but also at the construction phase and during the lifetime of the building.

In this paper these questions ane critically revised, focusing in the analysis of the main differences between the old and new codes, and the degree of convergence of the latter with the future European Code in this field (Eurocode 8).

\section{Introducción}

La actual Norma Española de Construcción Sismorresistente NCSE-94 fue aprobada por el Real Decreto 2543/1994 de 29 de diciembre (BOE del 8 de febrero de 1995), a iniciativa de la Comisión Permanente de Normas Sismorresistentes y ha venido a sustituir en el tiempo a la Norma Sismorresistente PDS-1 (1974),
Parte A, aprobada por el Decreto 3209/1974 de 30 de agosto.

De acuerdo con las Disposiciones Transitorias del Real Decreto, el día 8 de febrero de 1997 expiró el plazo de aplicación simultánea de ambas normas, la nueva y la antigua, esta última derogada hace ahora poco más de dos años. 
La norma PDS-1 (1974) fue promulgada para elevar a definitiva la primera norma española de construcción sismorresistente, la PGS-1, aprobada con carácter provisional en 1968. Esta última norma contenía dos capítulos específicamente dedicados a obras civiles, los cuales han desaparecido en la normativa subsiguiente que se extiende únicamente al ámbito de la edificación.

En los veinte años transcurridos desde la promulgación de la norma de 1974 la Comisión ha procedido a actualizar el Código Sismorresistente, adecuando las premisas básicas del mismo a la evolución del estado del conocimiento y corrigiendo los fallos y deficiencias que han ido detectándose como consecuencia de su aplicación. Fruto de este importante trabajo ha sido la Norma NCSE, no exenta de críticas y polémicas en su articulado, pero cuya filosofia general y los indudables avances metodológicos que supone no pueden ni deben ignorarse. Dichos avances y las directrices conceptuales del nuevo texto normativo se exponen a continuación.

\section{Criterios de control y de aplicación de la Norma NCSE-94}

La nueva normativa es bastante más estricta quela anterior en lo referente al control sobre su aplicación. Este hecho responde al sentimiento generalizado entre los profesionales del tema de que las normas sísmicas anteriores, PGS-1 (1968) y PDS-1 (1974), no eran excesivamente explícitas sobre el control administrativo del cumplimiento del articulado, originando, en muchos casos, carencias o insuficiencias de dicho cumplimiento. Por ello, la Norma NCSE-94, en su Capítulo V, establece taxativamente la necesidad de incluir en los proyectos en que proceda un apartado específico de la Memoria con el título de "Acciones Sísmicas", el cual será requisito necesario para el visado del Proyecto, así como para la tramitación, aprobación y expedición dela correspondiente licencia de obras (Artículo 5.1).

El control de aplicación de la Norma se extiende a las fases de construcción y explotación de la obra (Artículos 5.2 y 5.3), especificándose que es responsabilidad del director de obra cumplir con el apartado de Acciones Sísmicas o modificarlo (tras las oportunas aprobaciones) si estuviese en desacuerdo con el mismo. Además, si durante el período de vida útil de la obra se registrase un terremoto de intensidad igual o superior a VII (escala M.S.K), el técnico encargado de la conservación y explotación -o, en su defecto, la propiedad- deberá elaborar un informe en el que se analicen las consecuencias del sismo sobre la construcción y el tipo de medidas que proceda adoptar.

En cuanto a los criterios de aplicación de la Norma, se establecen en función de la "peligrosidad sísmica" o grado de exposición de la obra al riesgo de terremoto, la tipología constructiva y el material de construcción empleado. Estos factores aparecen interconectados entre sí por lo que se analizarán más adelante.

\section{Definición de las acciones sísmicas de proyecto}

Éste es uno de los aspectos en que más se distancia la filosofia de la nueva Norma respecto a la de la norma anterior, por dos razones fundamentales:

a) el concepto de peligrosidad como base de la información sísmica ha sustituido al de intensidad macrosísmica, representándose el riesgo anual de ocurrencia de terremotos en forma de mapas probabilísticos para un período de retorno fijo de la acción.

b) se considera en el articulado la definición del espectro de respuesta como modelización del movimiento sísmico del terreno a efectos de cálculo estructural.

La peligrosidad sísmica es un concepto puramente sismológico que se define como la probabilidad anual de que, por efecto de un terremoto de magnitud y localización aleatorias, se supere en el lugar un determinado parámetro representativo del nivel de excitación del terreno, normalmente la aceleración. Este concepto, objetivo y cuantificable, no debe confundirse con el de riesgo sísmico (concepto subjetivo ingenieril), que no es sino la distribución temporal y espacial del daño potencial a las estructuras.

Las isolíneas de peligrosidad, expresadas en términos de aceleración, se dibujan para todo el territorio nacional en el correspondiente mapa de la Norma (Fig. 1), que se ha confeccionado para una probabilidad anual de presentación del evento sísmico del $2 \%$.

\subsection{Aceleraciónsísmicade cálculo. Coeficiente de riesgo}

El mapa de peligrosidad y el Anejo 1 de la Norma especifican, para cada término municipal del Estado Español situado en una zona potencialmente sísmica, la denominada "aceleración sísmica básica", $a_{b}$, esto es, un valor característico de la aceleración horizontal de la superficie del terreno correspondiente a un período de retorno de referencia de 500 años. Obviamente, el valor del período de retorno es función de la intensidad o aceleración sísmica adoptada en el cálculo, por lo que la Norma, para evitar dar una colección de mapas de peligrosidad con distintos períodos de retorno dela acción, opta por definir un segundo nivel de aceleración, que depende no sólo de la ocurrencia del terremoto sino de la importancia de la construcción y la vida en servicio de ésta. Esta aceleración se denomina "aceleración sísmica de cálculo", $a_{c}$, y se relaciona con la aceleración sísmica básica del modo siguiente (Fig. 2):

$$
a_{c}=\rho a_{b}
$$




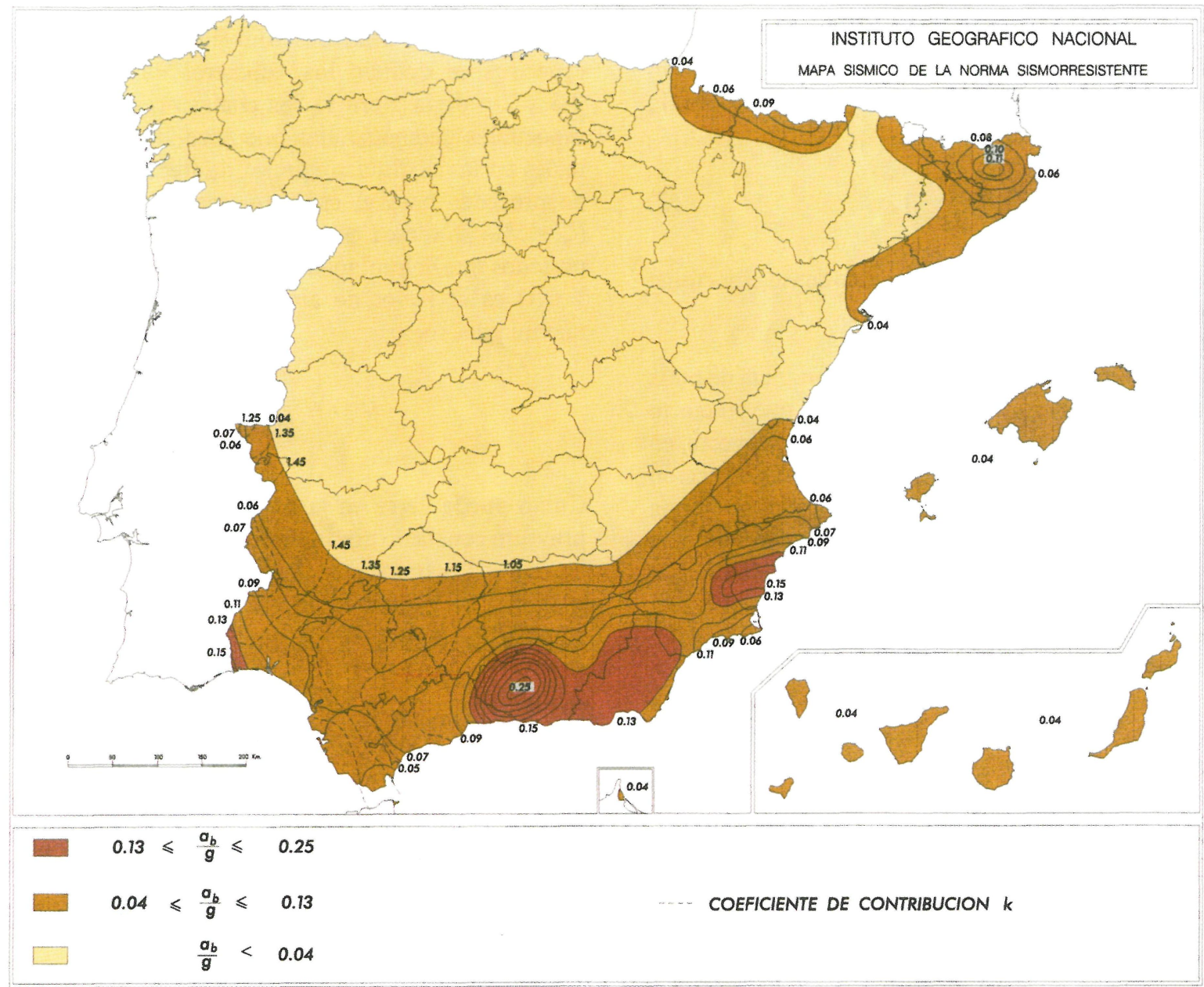

Fig. 1.- Mapa de peligrosidad sísmica de la Norma NCSE-94.

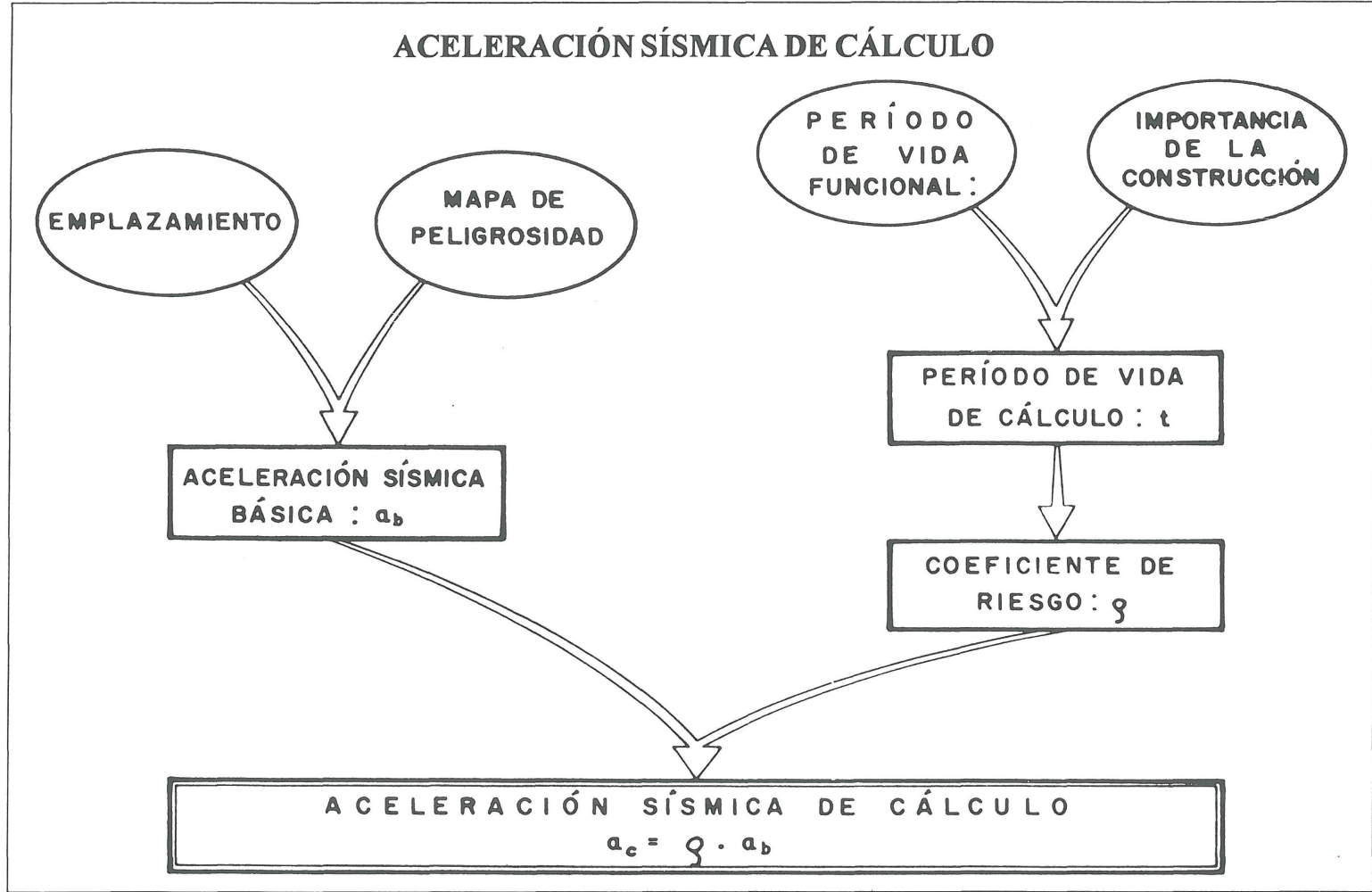

Fig. 2.- Esquema de la obtención de la aceleración sísmica de cálculo (Martín, 1992). 
donde $\rho$ es un coeficiente adimensional denominado "coeficiente de riesgo", cuyo valor viene dado en función del período de vida en años, $t$, para el que se proyecta la construcción:

$$
\rho=\left(\frac{t}{50}\right)^{0,37}
$$

que, en general, será mayor que el período de vida funcional de la obra.

El factor $\rho$ equivale al coeficiente de importancia de otras normas (el Eurocódigo 8, por ejemplo), ya que su efecto es mayorar la aceleración básica en la fase de proyecto, en función del uso, destino o importancia de la construcción $y$, por tanto, de su período de vida. De hecho, la Norma NCSE-94 liga el valor de $\rho$ a la importancia de la construcción (Art. 1.2), que se clasifica cualitativamente en una escala de tres grados de importancia: moderada, normal y especial (TABLA I). Así, para construcciones de especial importancia ( $t \geq 100$ años) se obtiene $\rho=1,3$, lo que equivale, en la práctica, a considerar en el proyecto la aceleración sísmica que posee un período de retorno de 1.000 años.

\subsection{Espectros elásticos de respuesta}

Constituyen la forma usual de caracterizar los movimientos sísmicos del terreno cuando se utiliza el cálculo modal para el análisis lineal de la estructura. Los espectros de respuesta reales no son otra cosa que gráficos frecuenciales en los que se representan las respuestas máximas, en aceleraciones absolutas, de una serie de osciladores simples, con distintos períodos propios y el mismo nivel de amortiguamiento, sometidos en su base al movimien- to sísmico que se desea describir (Fig. 3). A partir de estos espectros se definen los "espectros de proyecto" (Art 2-3 y 2-4 de la Norma), los cuales no son sino formas espectrales geometrizadas que aproximan los espectros reales en las distintas regiones de frecuencias. La forma de proceder para el espectro horizontal de referencia $(\zeta=5 \%)$ se indica en la Fig. 4 , donde $\mathrm{T}_{0}$ y $\mathrm{T}_{1}$ son los períodos que delimitan las distintas regiones del espectro y $\alpha(t)$ es el factor de amplificación de la aceleración máxima del suelo:

$$
\alpha(t)=\frac{S_{a}}{[a(t)]_{\text {máx. }}}
$$

Como puede verse en la Fig. 4, el espectro elástico de proyecto consta de tres tramos, a saber: tramo de períodos bajos ( $\left.\mathrm{T}<\mathrm{T}_{0}\right)$, tramo de períodos intermedios $\left(\mathrm{T}_{0} \leq \mathrm{T} \leq \mathrm{T}_{1}\right)$ y tramo de períodos altos $\left(\mathrm{T}>\mathrm{T}_{1}\right)$, con factores de amplificación respectivos:

$\begin{array}{ll}\alpha(\mathrm{T}) \div \mathrm{T} & \text { (estructuras rígidas) } \\ \alpha(\mathrm{T})=\alpha\left(\mathrm{T}_{0}\right) & \text { (estructuras intermedias) } \\ \alpha(\mathrm{T}) \div 1 / \mathrm{T} & \text { (estructuras flexibles) }\end{array}$

El conocimiento del período propio de vibración de la estructura resulta fundamental a la hora de evaluar su comportamiento sísmico, ya que los parámetros $T_{0}, T_{1}$ y $\alpha\left(\mathrm{T}_{0}\right)$ dependen del tipo de terreno (factor de suelo $\mathrm{C}$ ) y la filosofía de la Norma es evitar fenómenos de resonancia, alejando lo más posible el período fundamental de la estructura del período predominante del suelo de cimentación. Así, por ejemplo, estructuras flexibles cimentadas sobre suelo blando tipo $\mathrm{III}(\mathrm{C}=1,8)$ o estructuras rígidas cimentadas sobre suelo firme tipo $I(C=1,0)$

TABLA I

Clasificación de las construcciones según su grado de importancia (Martín, 1992)

\begin{tabular}{|c|c|c|c|}
\hline \multirow{2}{*}{ CLASES } & \multicolumn{3}{|c|}{ CR I TER I O S } \\
\cline { 2 - 4 } & VÍCTIMAS & $\begin{array}{c}\text { DAÑOS } \\
\text { ECONÓMICOS } \\
\text { A TERCEROS }\end{array}$ & $\begin{array}{c}\text { INTERRUPCIÓN } \\
\text { DE SERVICIOS } \\
\text { PRIMARIOS }\end{array}$ \\
\hline IMPORTANCIA & PROBABILIDAD DESPRECIABLE \\
MODERADA & EFECTOS NO CATASTRÓFICOS & $\begin{array}{c}\text { SERVICIOS } \\
\text { NO }\end{array}$ \\
IMPORTANCIA & IMPRESCINDIBLESS \\
\hline ESPECIAL & EFECTOS CATASTRÓFICOS & SERVICIOS \\
IMPORTANCIA & IMPRESCINDIBLES \\
\hline
\end{tabular}




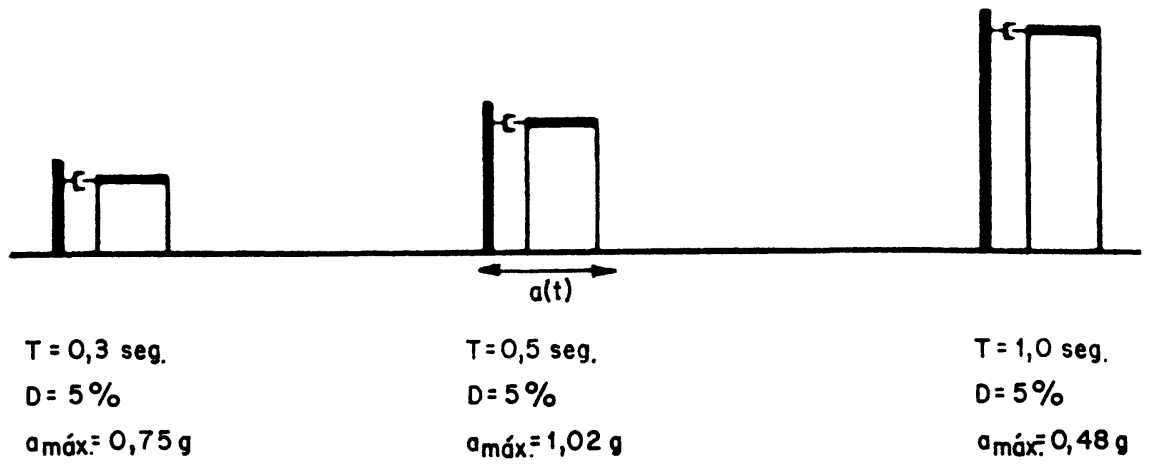

$a(t) / g$

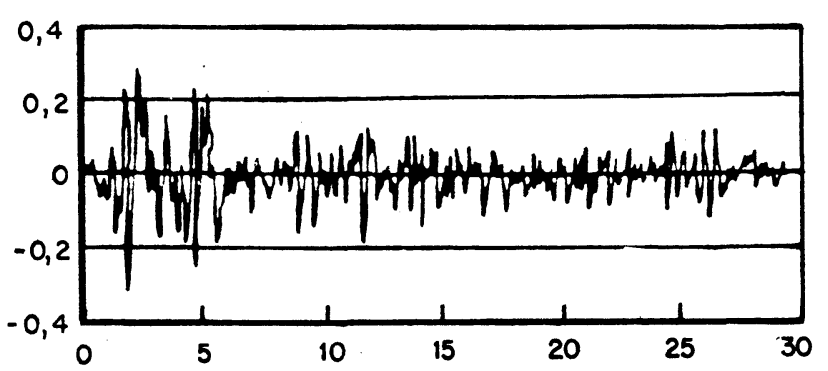

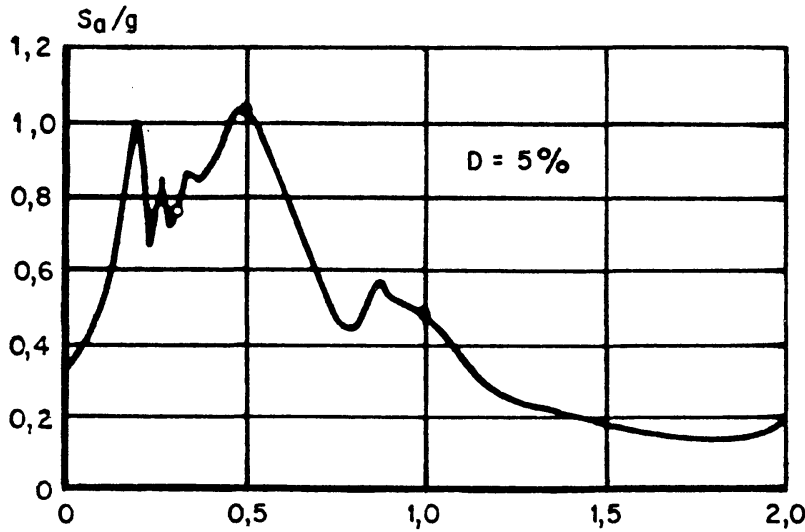

Fig. 3.- Definición del espectro de respuesta de un acelerograma.

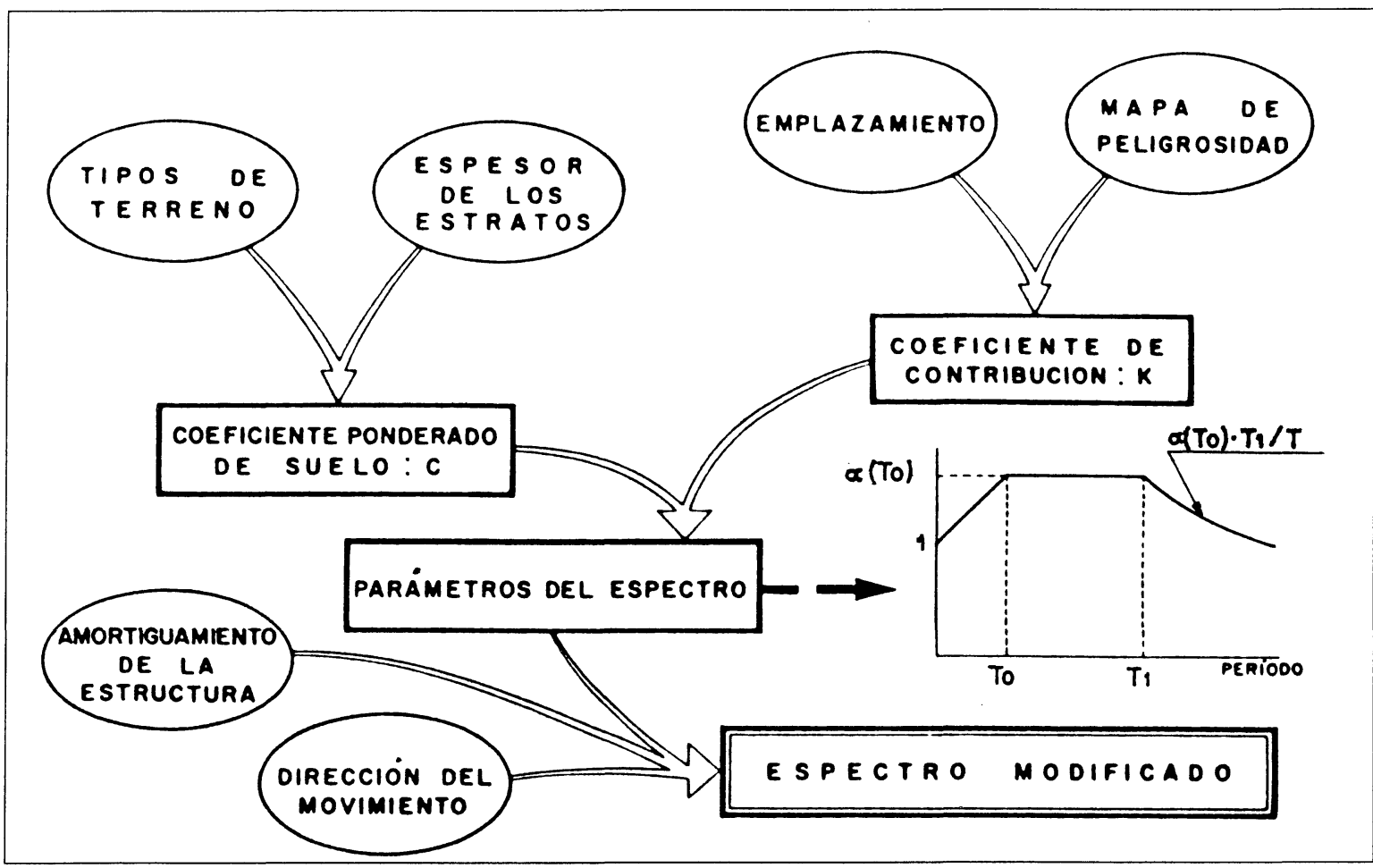

Fig. 4:- Obtención del espectro horizontal de proyecto según la Norma NCSE-94 (Martín, 1992).

(c) Consejo Superior de Investigaciones Científicas 
resultan ser altamente vulnerables en caso de terremotos (Fig. 5-a)

Los parámetros $\mathrm{T}_{0}, \mathrm{~T}_{1} \mathrm{y} \alpha\left(\mathrm{T}_{0}\right)$ dependen también del tipo de terremoto, esto es, del mecanismo focal y de la posición relativa del emplazamiento con relación a la fuente sismogenética. Para ello la Norma define un coeficiente $\mathrm{K}$, denominado coeficiente de contribución (líneas discontinuas en el mapa dela Fig. 1), que tiene en cuenta la distinta participación en el cómputo de la peligrosidad sísmica global en un determinado lugar de los terremotos peninsulares próximos $(\mathrm{K} \rightarrow 1)$ y de los más lejanos $(\mathrm{K} \rightarrow 1,5)$. Estos últimos, generados en la falla AzoresGibraltar, poseen mayor magnitud, menor atenuación con la distancia y un mayor contenido de bajas frecuencias (Fig. 5-b).
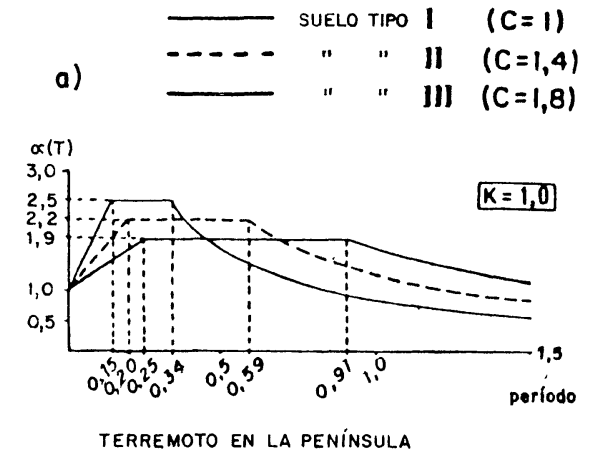

Fig. 5.- Variación de las formas espectrales con el tipo de terremoto y la resistencia mecánica del terreno (Norma NCSE-94).

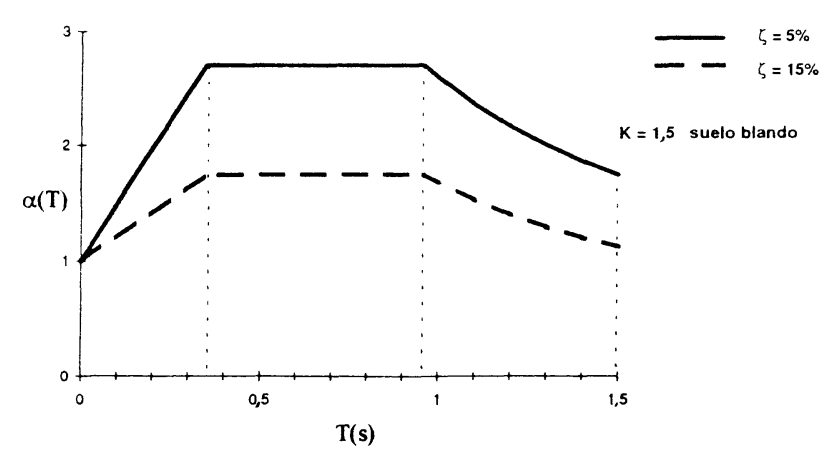

Fig. 6.- Ejemplo de espectros de proyecto de movimientos horizontales para distintos amortiguamientos (Blázquez, 1995).

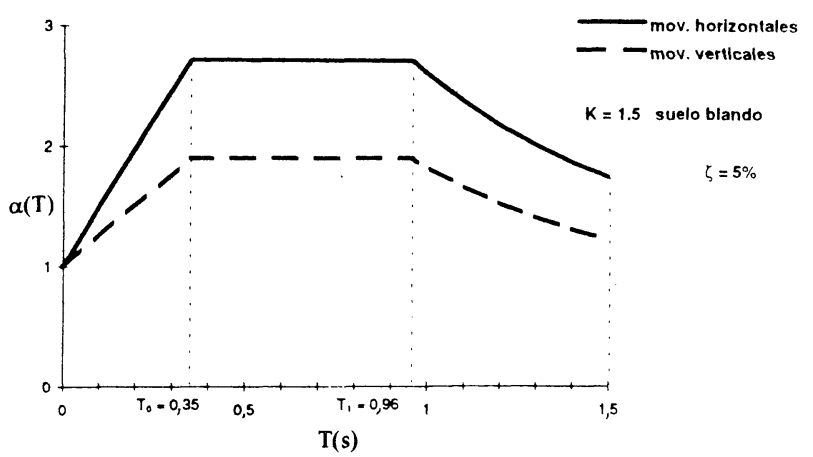

Fig. 7.- Ejemplo de espectros de proyecto de la Norma NCSE-94 para movimientos horizontales y verticales (Blázquez, 1995).
La Norma especifica, asimismo, la forma de modificar el espectro horizontal de referencia en función del amortiguamiento (multiplicando por el factor $v=(5 / \zeta)^{0,4}$, Fig. 6) y la forma de derivar, a partir de aquél, el espectro elástico de respuesta para movimientos verticales (multiplicando por el factor 0,7; Fig. 7).

Es interesante constatar que los espectros de proyecto proporcionados por la Norma rebajan bastante las cargas sísmicas en áreas epicentrales, por comparación con los espectros reales de acelerogramas registrados en dichas zonas. Esta observación es aplicable tanto a movimientos relativamente débiles del terreno (Fig. 8), como a movimientos fuertes (Bertero, 1989) y concuerda con las informaciones macrosísmicas disponibles en diversas partes del mundo. b)

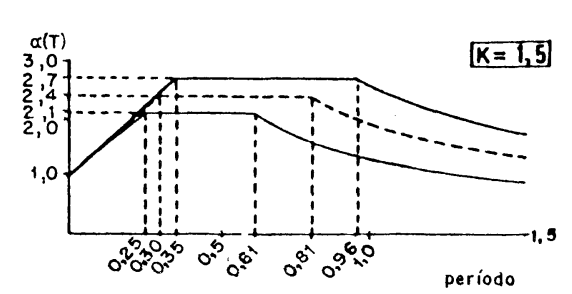

TERREMOTO EN LA FALLA AZORES-GIBRALTAR

\section{$\zeta=5 \%$}


TABLA ॥

Representación esquemática de los criterios de aplicación de la Norma (Martín, 1992)

\begin{tabular}{|c|c|c|c|c|c|}
\hline \multirow{2}{*}{\multicolumn{2}{|c|}{$\begin{array}{c}\text { CLASE Y TIPO } \\
\text { DE } \\
\text { CONSTRUCCIÓN }\end{array}$}} & \multicolumn{4}{|c|}{ ACELERACIÓN SÍSMICA DE CÁLCULO } \\
\hline & & $<0,06$ & $0,06-0,08$ & $0,08-0,12$ & $>0,12$ \\
\hline \multirow{3}{*}{$\begin{array}{l}\text { IMPORTANCIA } \\
\text { ESPECIAL } \\
\text { O }\end{array}$} & $\begin{aligned} & \text { FÁBRICA DE } \\
& \text { - LADRILLOS } \\
& \text { - BLOQUES } \\
& \text { - } \\
& \text { ETC. }\end{aligned}$ & \multirow{3}{*}{$\begin{array}{c}\text { NORMA } \\
\text { NO } \\
\text { OBLIGATORIA }\end{array}$} & $\begin{array}{l}\text { SIN } \\
\text { LIMITACIÓN } \\
\text { EN ALTURA }\end{array}$ & $\begin{array}{l}\text { MÁXIMO } \\
\text { CUATRO } \\
\text { ALTURAS }\end{array}$ & $\begin{array}{l}\text { MÁXIMO } \\
\text { DOS } \\
\text { ALTURAS }\end{array}$ \\
\hline & $\begin{array}{c}\text { MAMPOSTERIA } \\
\text { EN SECO }\end{array}$ & & \multicolumn{3}{|c|}{ PROHIBIDA } \\
\hline & $\begin{array}{l}\text { HORMIGÓN } \\
\text { ARMADO, } \\
\text { METÁLICAS Y } \\
\text { SIMILARES }\end{array}$ & & \multicolumn{3}{|c|}{ SIN LIMITACIÓN EN ALTURA } \\
\hline $\begin{array}{l}\text { IMPORTANCIA } \\
\text { MODERADA }\end{array}$ & & \multicolumn{4}{|c|}{ NORMA NO OBLIGATORIA } \\
\hline
\end{tabular}

En la Tabla II se matizan estas cuestiones, tanto a lo referente al ámbito territorial de obligado cumplimiento de la Norma $\left(\mathrm{a}_{\mathrm{c}}>0,06 \mathrm{~g}\right)$ como al grado de importancia (normal o especial) de las construcciones afectadas.

Cuando el cálculo sísmico es obligado, la norma permite tres opciones para el cálculo estructural con base fija (el aislamiento de base no está contemplado en la Norma):

a) método simplificado

b) análisis modal espectral

c) estudio dinámico directo

El método simplificado, que se utiliza en la Norma PDS-1(1974), se permite para los casos más usuales de edificación, esto es, para construcciones asimilables a osciladores múltiples con un grado de libertad por planta. Consiste, en esencia, en el método pseudoestático clásico al que se han puesto una serie de restricciones que no figuraban en la anterior norma, tales como máxima altura permisible $(60 \mathrm{~m})$ o máximo número de plantas $(20)$, regularidad geométrica y mecánica del edificio en planta y en alzado (masas, rigideces y resistencias), excentricidad del centro de masas respecto al de torsión inferior al $10 \%$ de la máxima dimensión en planta, etc.

El análisis modal espectral se establece como método general para el cálculo de edificaciones. Está basado en el espectro de respuesta, permite varios grados de libertad por planta y requiere la combinación ponderada de las solicitaciones provenientes de cada modo de vibración de la estructura (reglas SRSS o CQC, según que los períodos modales difieran en más o menos de un $10 \%$ ).

Una de las novedades incorporadas por la nueva Norma es la introducción del "coeficiente de comportamiento por ductilidad de la estructura", $\mu$, el cual se combina con el coeficiente $\mathrm{v}$ de modificación del amortiguamiento estándar $(5 \%)$ en el denominado "coeficiente de respuesta":

$$
\beta=v / \mu
$$

Este coeficiente modifica la acción sísmica para adecuarla a la respuesta dela estructura, caracterizada por su grado de compartimentación, su amortiguamiento viscoso y su ductilidad en régimen plástico. En congruencia con el significado de $\mu$, el cálculo sísmico preconizado por la Norma supone realizar un análisis elástico para una aceleración equivalente a la aceleración de cálculo dividida por el coeficiente de comportamiento por ductilidad. El valor máximo del parámetro $\mu$ se limita a 4 , de forma que las construcciones de normal importancia resistan en régimen elástico los sismos con período de retorno de 50 años, es decir, los quetienen una probabilidad apreciable de ocurrencia durante el período de vida de la estructura (estado límite de servicio). Entonces, tan sólo para los sismos de intensidad fuerte $\left(\mathrm{T}_{\text {ret }}>50\right.$ años $)$ que ocurran en el período de vida útil de la estructura se producirán incursiones de ésta en el dominio elastoplástico.

El estudio dinámico directo, realizado en el dominio del tiempo o de la frecuencia, se permite siempre que los acelerogramas utilizados (mínimo tres) sean compatibles con la información sísmica básica del emplazamiento. En el caso de acelerogramas reales, los valores característicos de los esfuerzos o desplazamientos deben obtenerse aplicando un factor de mayoración de 1,35. Si se emplean acelerogramas artificiales, las desviaciones máximas permisibles, respecto a las ordenadas espectrales de proyecto, son del $10 \%$. 